\title{
Caries y otras condiciones de la boca de niñas escolares de diferente nivel económico y social $^{*}$
}

por

Raymond Pauly S.**:

(Recibido para su publicación el 15 de noviembre de 1955)

En los años recientes se le viene concediendo en Costa Rica, la debida importancia a la atención dental del niño, aún cuando los estudios efectuados al respecto son muy pocos. De ellos pueden mencionarse, el de SCRimshaw et al. (13) (21), en la zona de Turrialba, en el que se determinó el $\mathrm{DMF}^{1}$ de 137 familias, y el del Departamento de Higiene Dental del Ministerio de Salubridad Pública, en el cual se examinaron 3022 niños de veinticinco escuelas primarias de San José, con el propósito de determinar la acción reductora de caries en años posteriores, con la fluorinación del agua de consumo iniciada en 1952 (17).

En 1954, el Departamento de Odontología Infantil y Preventiva de la Facultad de Odontología de la Universidad de Costa Rica, realizó una "Campaña Educativa pro-Salud Oral del Niño", en las escuelas primarias de la capital, que posteriormente, con la colaboración de la Facultad de Pedagogía, fue posible extender a toda la provincia de San José. Esto se hizo por medio de láminas educativas, tomando como base los grabados de la Asociación Dental Americana, pero con los textos de las mismas, adaptados al medio costarricense. Sin embargo, dicha campaña fue planeada en términos generales, llegándose luego a la conclusión básica, de la necesidad de efectuar un estudio estadístico, que aunque en pequeña escala, permitiera formarse un idea de las

* Trabajo presentado al III Congreso Dental Nacional de México, octubre de 1955.

* Cátedra de Odontología Infantil y Preventiva de la Facultad de Odontología de la Universidad de Costa Rica.

1 Por DMF se entiende el índice que expresa la experiencia acumulada de caries. $\mathrm{D}=$ decay; $\mathrm{M}=$ missing; $\mathrm{F}=$ filling. 
condiciones de major prevalencia en los niños costarricenses, a efecto de poder orientar su futura educación dental en una forma concreta.

Hasta el momento, los estudios representan la mitad del plan completo, o sea los efectuados en los grupos femeninos, que aún cuando no permitan llegar a conclusiones del todo definitivas, dan una buena idea de las condiciones investigadas, y sobre todo, de las diferencias existentes entre grupos de distinta condición económica y social, pudiéndose interpretar este último término, como el aspecto de educación.

Por consiguiente, el propósito fundamental de este trabajo es, a la vez que el de dar a conocer la importancia que se le concede en la actualidad a la atención dental del niño, en los círculos profesional, sanitario y estudiantil de Costa Rica, el de exponer los métodos seguidos en esta investigación y las conclusiones básicas obtenidas.

\section{MATERIAL Y METODO}

El estudio incluye niñas de diferentes esferas sociales y económicas, condiciones estas últimas, de gran consideración, como se verá oportunamente. Las muestras se tomaron de dos Escuelas: la República de Chile, a la cual asisten alumnas de mala condición económica e hijas de la clase obrera y la República del Perú que es aquella escuela de San José donde se encuentras las niñas de mejores recursos económicos y cuyos padres son en su mayoría, profesionales, comerciantes $e$ industriales. Los resultados obtenidos de este estudio se encuentran clasificados y tabulados.

La edad escolar en Costa Rica se encuentra comprendida entre los siete y los trece años, y el plan de estudios comprende seis grados. Se tomó un total de 360 muestras, que encierran 60 de cada 6 dad, divididas a su vez, en 30 de cada uno de los grupos de distinto nivel económico y social. Se denominó a las niñas de la Escuela República de Chile, como grupo A, y a las de la Escuela República del Perú, como grupo B. Por las ligeras variaciones en la edad de las niñas en cada grado escolar, se clasificaron en la siguiente forma:

$\begin{array}{rrrrlllll}\text { De } & 7 & \text { a } & 8 & \text { años } & \text { Grupo } & 1-\mathrm{A} & \text { Grupo } & 1-\mathrm{B} \\ \text { De } & 8 & \text { a } & 9 & \text { años } & \text { Grupo } & 2-\mathrm{A} & \text { Grupo } & 2-\mathrm{B} \\ \text { De } & 9 & \text { a } & 10 & \text { años } & \text { Grupo } & 3-\mathrm{A} & \text { Grupo } & 3-\mathrm{B} \\ \text { De } & 10 & \text { a } & 11 & \text { años } & \text { Grupo } & \text { 4-A } & \text { Grupo } & 4-\mathrm{B} \\ \text { De } & 11 & \text { a } & 12 & \text { años } & \text { Grupo } & 5-\mathrm{A} & \text { Grupo } & 5-\mathrm{B} \\ \text { De } & 12 & \text { a } & 13 & \text { años } & \text { Grupo } & 6-\mathrm{A} & \text { Grupo } & 6-\mathrm{B}\end{array}$

Se presentó el inconveniente, de que muchas de las niñas del grupo A son repitentes, no guardando relación con la edad del grado correspondiente, por lo que al final hubo que descartar once muestras, quedando el total reducido a 349 .

Antes de proceder el examen clínico de la boca y de los dientes, las niñas fueron minuciosamente interrogadas por los instructores del Departamento de Odontología Infantil y Preventiva, con la coĺaboración de sus maestras, a efecto 
de lograr la mayor exactitud en los datos que se encuentran especificados en la hoja clínica, y que pueden explicarse en la siguiente forma:

\section{Datos de encabezamiento}

Nombre, edad, escuela, grado, sexo, constitución y raza (puntos que no demostraron tener influencia en la aparición o no de caries). Condición económica y nivel de educación social. Maestro (a), dato importante para localizar cualquier niña que por alguna circunstancia debiera ser reexaminada.

\section{Estado de la boca}

Esta condición se determinó después del examen clínico, de acuerdo con el criterio del instructor y del estudiante, y tomando en consideración: el número de caries, el progreso de las mismas, estado de las obturaciones, piezas extraídas, piezas por extraer, restos radiculares, estado de higiene, condición de los tejidos blandos, etc. En esas bases, cada muestra se incluyó en uno de los siguientes grupos: Buena, aceptable, regular, mala, pésima.

\section{Consumo de azúcar}

Se quiso determinar aproximadamente la cantidad que consume cada niña diariamente, tanto en sus horas escolares, como junto con sus comidas. Hubo que admitir sin embargo, que es una de las condiciones más difíciles de determinar en un niño (14), ya que dan una información falsa, o bien, niegan consumirlo. Sí pudo comprobarse, que en ambos grupos el consumo de golosinas es frecuente en horas escolares y junto con sus comidas, así como más elevado en el grupo A, ya que en su hogar, estos niños sustituyen la leche, por el "agua dulce" y el café con azúcar.

\section{Frecuencia de la visita al dentista}

Aquí se consideraron cinco puntos: Privado, dispensario público, por rutina, por emergencia, nunca. En determinar las dos primeras circunstancias no hubo dificultad. Sin embargo, en la visita por rutina, hubo que consideras más bien aquellas niñas, que aún cuando no asisten periódicamente donde el dentista, han sido llevadas donde el mismo en repetidas ocasiones, para atención de su boca. Por la condición de los dientes, complementado con el interrogatorio de la niña, fue fácil establecer las que nunca lo han visitado.

\section{Higiene de la boca}

Con la ayuda de la maestra, se indagó sobre los recursos de las niñas para efectuar el cepillado de sus dientes, considerando si disponian o no de 


\section{UNIVERSIDAD DE COSTA RICA \\ FACULTAD DE ODONTOLOGIA \\ DEPARTAMENTO DE ODONTOLOGIA INFANTIL Y PREVENIIVA}

\section{EXAMEN ORAL DE ESCOLARES DE SAN JOSE}

Nombre

Edad

Escuela

Grado.

Sexo

Constitucion

Ruza

Condición Eiconónica

Nivel de Educación Social

Maestro (a)

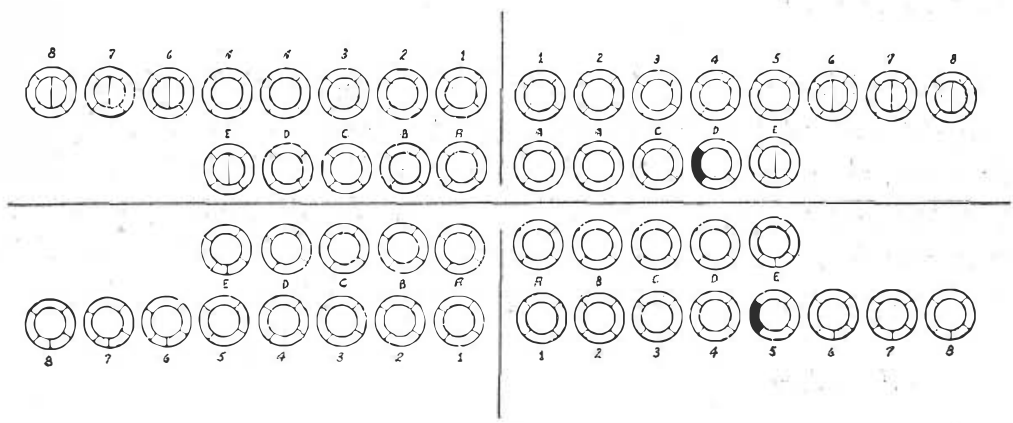

1 - estado de la boca

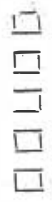

\section{Buena}

Aceptable

Regular

Mala

Pesima
2 - CONSUMO de AzUCAR

II Noda

El Ocosicnal

II Frecuente

-1. Excesivo

4. HIGIENE DE SU BOCA

I. Poseecepillo I Si No $_{\text {II Por pobreza }}\left\{\begin{array}{l}\text { I Por negligencia } \\ \text { I Psa dentífrico I Sí }\end{array}\right.$ No $\left\{\begin{array}{l}\text { II Por pobreza } \\ \text { II Por negligencia }\end{array}\right.$ III. Se cepilla los dientes: I Nunca I Uno vez al día $\square$ Dos veces al día I Tres veces al día II Más veces al día I Antes del desayuno I Después del desayuno II Antes de las comidas I Después de las comidas II Antes de acostarse.

\section{NUMERO DE CARIES}

Examinado por

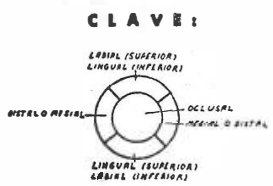

INDICE C. O. E.

Vo. Bo.
Coriodar Lapiz nojo

Obiuroda: Lapiz Azul

Exiroídat $x$

Campaña Educativa pro Salud Oral, dirigida por el Departamento de Odontologia_Infantil y Preventiva de la Facultad de Odontologia 
cepillo y dentífrico, $y$ en este último caso, si por pobreza o por negligencia. La información obtenida fue bastante satisfactoria. Sin embargo, en lo referente a la hora y al número de veces al día que efectúan la limpieza de la boca, se comprobó al hacer el examen de la misma, que los datos no coincidían, optándose por no tomar en consideración dicho punto.

Obtenidos los datos anteriores, cada niña fue examinada clínicamente por estudiantes de años superiores, y luego $\epsilon$ l caso revisado por un instructor. Para un resultado más efectivo el examen se hizo en el siguiente orden: cuadrante superior derecho, comenzando por la última molar; cuadrante superior izquierdo, continuando por el central del mismo lado, cuadrante inferior izquierdo y cuadrante inferior derecho. En cada pieza se exploraron cuidadosamente todas sus superficie: susceptibles.

Como lo demuestra el diagrama contenido en la hoja clínica, se consideraron por aparte, la dentición permanente y la dentición temporal, anotando en cada una de ellas, una serie de condiciones, con la siguiente clave para facilitar la lectura de las mismas: Caries (punto rojo en la superficie afectada). Obturada (punto azul en la superficie tratada). Extraída (X, en rojo). Por extraer (/, en rojo). Exfoliada (E).

Lógicamente resulta difícil determinar un def ${ }^{1}$ (8) exacto en la dent:ción mixta, por ignorarse en muchos casos si la pieza temporal se exfolió o fue extraída, y en consecuencia, para una major aproximación se tomaron en cuenta las siguientes consideraciones: Edad; la interrogación a la niña, sobre si ha pieza se desprendió o fue extraída; la pérdida o no del espacio, la erupción anticipada de la premolar; la existencia o no de la antagonista o de la del lado opuesto.

Finalmente se procedió, de acuerdo con lo ya expuesto, a clasificar la condición de la boca y a anotar cualquier tipo de mala oclusión presente, que por el reducido número de casos, no se contemplarán todavía en este estudio.

Clasificadas las fichas en las edades correspondientes se determinó en cada niña, en cada sub-grupo, en cada grupo y en un término promedio de ambos grupos, las siguientes condiciones de los dientes:

\section{A. Dentición permanente}

1. El DMF (Knutson, Klein y Palmer) $(8,16)$, pero para mejor interpretación, traducido al castellano, como COE (20) (piezas cariadas = C; obturadas $=\mathrm{O}$; extraídas $=\mathrm{E}$ ). Este índice se determinó, tanto por pieza dentaria, como por superficie afectada en cada pieza, en cuyo caso se atribuyó, de acuerdo con lo acostumbrado por muchos investigadores (14), un valor de tres puntos a las piezas extraídas. 
2. El número de caries, sin considerar piezas obturadas y extraídas.

3. El número de obturaciones, a efecto de poder comprobar con el dato anterior, el porcentaje de piezas cariadas que requieren tratamiento.

4. El índice de salud dental (Turner, How y Dick) (20) (15), referente al número de primeros molares permanentes perdidos.

5. El indice de mortalidad dentaria (Knutson y Klein) (20) (15), que se refiere al número de piezas permanentes perdidas, incluyendo las primeras molares.

6. El número de caries por superficie, considerando, oclusales, proximales y de otros tipos. En este punto, se determinó tanto el promedio de cada tipo por niño, como la relación o proporción que guardan entre ellas.

7. Indice de afección del primer molar permanente.

\section{B. DenTICIÓN TEMPORAL}

1. El def (Gruebel) (20), igualmente traducido al castellano como coe (minúsculas), determinándolo tanto por pieza, como por superficie.

2: Número de caries.

3. Número de obturaciones. Ambos puntos con el propósito expresado anteriormente.

4. Número de caries por superficic.

5. Mortalidad dentaria temporal.

Los resultados en ambas denticiones, se ordenaron en cuadros, tanto comparativos entre ambos grupos, como del término promedio de ellos. Las condiciones contenidas en ambos cuadros se expresan en forma de gráficas, que se discutirán oportunamente. Se tabularon también en forma comparativa, los datos obtenidos sobre la condición de la boca, la frecuencia de las visitas al dentista y los recursos para la higiene de la boca.

\section{DISCUSION}

En el estudio minucioso de los cuadros y de los gráficos se pueden observar una serie de condiciones importantes, que se discuten a continuación:

\section{Cuadro 1:}

Expresa los términos promedios de las condiciones estudiadas en la dentición permanente, en una forma comparativa entre ambos grupos, que en las gráficas correspondientes dan una mejor idea del asunto. Cabe sólo discutir dos puntos de este cuadro.

1?-El índice de mortalidad dentaria entre los 10 a los 13 años, lógicamente se manifiesta más elevado que el de salud dental, pero la diferencia entre ambos es mínima, no justificándose su expresión en forma gráfica. Se manifiesta considerablemente más elevado en las niñas del grupo A. 


\section{CUADRO 1}

Cuadro comparativo de las condiciones estudiadas en los dientes de dos grupos de niñas escolares de 7 a 13 años.- 350 muestras

DENTICION PERMANENTE

\begin{tabular}{|c|c|c|c|c|c|c|c|c|c|c|c|}
\hline \multirow{2}{*}{$\begin{array}{c}N^{\prime \prime} \\
\text { niños }\end{array}$} & \multirow{2}{*}{$\begin{array}{l}\text { Eclad y } \\
\text { grupo }\end{array}$} & \multirow{2}{*}{$\begin{array}{l}\text { C.e.E. } \\
\text { Super. } \\
\text { ficie }\end{array}$} & \multirow{2}{*}{$\begin{array}{c}\text { C.O.E. } \\
\text { Fieza }\end{array}$} & \multirow{2}{*}{$\begin{array}{c}\text { N? } \\
\text { Caries }\end{array}$} & \multirow{2}{*}{$\begin{array}{c}\mathrm{N}^{\prime \prime} \\
\text { Obtura. } \\
\text { ción }\end{array}$} & \multirow{2}{*}{$\begin{array}{l}\text { Salud! } \\
\text { Dental }\end{array}$} & \multirow{2}{*}{$\begin{array}{c}\text { Morta!i- } \\
\text { dad } \\
\text { dentaria }\end{array}$} & \multicolumn{3}{|c|}{ Caries: Superficial } & \multirow{2}{*}{$\begin{array}{c}\text { Indice } \\
\text { afec- } \\
\text { ciones } \\
\text { primer } \\
\text { molar }\end{array}$} \\
\hline & & & & & & & & $\underset{\text { sal }}{\text { Oclu- }}$ & $\begin{array}{c}\text { Próxi- } \\
\text { má }\end{array}$ & Otras & \\
\hline 28 & $\begin{array}{c}7-8 a \\
1-A\end{array}$ & 6,3 & 3,8 & 6,0 & 0,2 & $\begin{array}{c}0 \\
0 / n\end{array}$ & $\begin{array}{c}0 \\
0 / n\end{array}$ & $\begin{array}{l}4,6 \\
1,5\end{array}$ & $\begin{array}{l}0,3 \\
1\end{array}$ & $\begin{array}{l}1,4 \\
5\end{array}$ & 3,5 \\
\hline 31 & $\begin{array}{c}7-8 \mathrm{a} \\
1 .-\mathrm{B}\end{array}$ & 5,6 & 3,2 & 5,1 & 0,1 & $\begin{array}{c}2 \\
0 / n\end{array}$ & $\begin{array}{c}2 \\
0 / n\end{array}$ & $\begin{array}{l}3,8 \\
38\end{array}$ & $\begin{array}{l}0,1 \\
1\end{array}$ & $\begin{array}{l}1,4 \\
14\end{array}$ & 3,0 \\
\hline 31 & $\begin{array}{l}8-9 \text { a } \\
2-A\end{array}$ & 8,2 & 4,5 & 6,9 & 0,2 & $\begin{array}{c}12 \\
0,3 / n\end{array}$ & $\begin{array}{l}12 \\
0,3 / \mathrm{n}\end{array}$ & $\begin{array}{l}5,0 \\
10\end{array}$ & $\begin{array}{l}0,5 \\
1 .\end{array}$ & $\begin{array}{l}1,4 \\
3\end{array}$ & 3,9 \\
\hline 28 & $\begin{array}{c}8-9 a \\
2-B\end{array}$ & 6,7 & 3,8 & 6,2 & 0,2 & $\begin{array}{c}2 \\
0 / \mathrm{n}\end{array}$ & $\begin{array}{c}2 \\
0 / n\end{array}$ & $\begin{array}{l}4,3 \\
7\end{array}$ & $\begin{array}{l}0,6 \\
1\end{array}$ & $\begin{array}{l}1,5 \\
3\end{array}$ & 3,4 \\
\hline 27 & $\begin{array}{c}9-10 \\
3-A\end{array}$ & 9,9 & 5,4 & 8,1 & 0,5 & $\begin{array}{c}9 \\
0,3 / n\end{array}$ & $\begin{array}{c}9 \\
0,3 / n\end{array}$ & $\begin{array}{l}5,4 \\
3\end{array}$ & $\begin{array}{l}1,7 \\
1\end{array}$ & $\begin{array}{l}1,9 \\
1,1\end{array}$ & 3,6 \\
\hline 29 & $\begin{array}{c}9-10 \text { a } \\
3-B\end{array}$ & 7,0 & 4,5 & 4,6 & 1,5 & $\begin{array}{c}0 \\
0 / 12\end{array}$ & $\begin{array}{c}0 \\
0 / n\end{array}$ & $\begin{array}{l}5,1 \\
6\end{array}$ & $\begin{array}{c}0,8 \\
1\end{array}$ & $\begin{array}{l}1,4 \\
1,7\end{array}$ & 3,5 \\
\hline 27 & $\begin{array}{c}10-11 a \\
4-A\end{array}$ & 12,1 & 7,0 & 9,4 & 0,4 & $\begin{array}{c}17 \\
0,6 / n\end{array}$ & $\begin{array}{l}20 \\
0,7 / n\end{array}$ & $\begin{array}{l}6,4 \\
3\end{array}$ & $\begin{array}{l}2,0 \\
1\end{array}$ & $\begin{array}{l}1,5 \\
0,8\end{array}$ & 4 \\
\hline 31 & $\begin{array}{c}10-11 \\
4-B\end{array}$ & 7,4 & 6,2 & 6,4 & 1,5 & $\begin{array}{c}1 \\
0 / n\end{array}$ & $\begin{array}{c}1 \\
0 / n\end{array}$ & $\begin{array}{l}5,8 \\
3\end{array}$ & $\begin{array}{l}1,8 \\
1\end{array}$ & $\begin{array}{l}1,3 \\
0,7\end{array}$ & 3,6 \\
\hline 28 & $\begin{array}{c}11-12 a \\
5-A\end{array}$ & 1,5 & 8,9 & 1,3 & 0,3 & $\begin{array}{l}12 \\
0,4 / n\end{array}$ & $\begin{array}{l}13 \\
0,4 / n\end{array}$ & $\begin{array}{l}8,5 \\
3\end{array}$ & $\begin{array}{l}3 \\
1\end{array}$ & $\begin{array}{l}1,7 \\
0,6\end{array}$ & 3,8 \\
\hline 31 & $\begin{array}{c}11-12 \mathrm{a} \\
5-\mathrm{B}\end{array}$ & 1,2 & 7,7 & 5,5 & 3,5 & $\begin{array}{c}7 \\
0,2 / n\end{array}$ & $\begin{array}{c}7 \\
0,2 / 11\end{array}$ & $\begin{array}{l}6,4 \\
2,5\end{array}$ & $\begin{array}{l}2,6 \\
1\end{array}$ & $\begin{array}{l}2,1 \\
0,8\end{array}$ & 3,7 \\
\hline 29 & $\begin{array}{c}12-13 a \\
6-A\end{array}$ & 19,1 & 11,4 & 15,5 & 0,9 & $\begin{array}{l}25 \\
0,8 / n\end{array}$ & $\begin{array}{c}34 \\
1,1 / \mathrm{n}\end{array}$ & $\begin{array}{c}10,2 \\
3 .\end{array}$ & $\begin{array}{l}3,2 \\
1\end{array}$ & $\begin{array}{l}2,6 \\
0,8\end{array}$ & 4 \\
\hline 30 & $\begin{array}{c}12-13 \mathrm{a} \\
6-\mathrm{B}\end{array}$ & 12,3 & 8,4 & 7,4 & 2,5 & $\begin{array}{c}6 \\
0,2 / 11\end{array}$ & $\begin{array}{c}9 \\
0,3 / n\end{array}$ & $\begin{array}{l}6,9 \\
3,5\end{array}$ & $\begin{array}{l}2 \\
1\end{array}$ & $\begin{array}{l}1,4 \\
0,7\end{array}$ & 3,3 \\
\hline & & & & & & & & & & & - \\
\hline
\end{tabular}


2q-En los distintos tipos de caries, puede notarse que la oclusal es la más frecuente en la dentición permanente, tal como lo expresa Noyes (9). Sin embargo, la relación que guarda con la caries proximal es variable y no sigue una línea progresiva conforme avanza la edad del niño. Por otro lado, la caries proximal se manifiesta en este estudio, en una forma relativamente reducida, lo cual confirma una vez más, la necesidad del examen radiológico rutinario en la práctica del niño (6), (10).

CUAdRo 2:

Se refiere a los términos promedios de las condiciones estudiadas en la dentición temporal, del mismo modo, en una forma comparativa entre ambos grupos, que se encuentran también expresadas en las gráficas. Del estudio comparativo de los tipos de caries, se desprende que la proximal en relación con la oclusal, es mucho más elevada que en la dentición permanente, siendo casi iguales en muchos sub-grupos, y sobrepasando la primera a la segunda en el cuadro de 7-8 años, $1-B$, que aunque puede interpretarse como un accidente del grupo con un reducido número de muestras, confirma la enorme prevalencia de caries proximales en la dentición temporal (10).

\section{CuAdros 3 Y 4 :}

Representan los términos promedios de ambos grupos, de las condiciones estudiadas en la dentición permanente y temporal respectivamente. Por el reducido número de muestras, los resultados obtenidos no permiten establecer índices definitivos, pero al menos dan una buena idea de la prevalencia de los factores investigados en los niños costarricenses, expresados asímismo en las gráficas correspondientes:

\section{GRÁFica 1:}

COE y coe por pieza, comparativos entre los grupos A y B. Se nota mayor incidencia de caries en ambas denticiones, en el grupo A. Las curvas de la dentición permanente son paralelas entre ambos grupos, pero se acentúa en el grupo A conforme avanza la edad (entre los 7 y los 8 años expresa una diferencia de 0,5 por niña, en tanto que entre los 12 y los 13 llega a 2,0), condición que indica en las niñas de este grupo, la preservación de hábitos indeseables, como el mayor consumo de carbohidratos, higiene de la boca descuidada y negligencia en las visitas al dentista. Este factor aumenta considerablemente la mortalidad dentaria. Por otro lado, puede establecerse, que aún cuando el consumo de carbohidratos refinados, sea el factor determinante de la caries dentaria (9) (3), a la falta de higiene debe concedérsele bastante importancia, como factor coadyuvante. El índice coe, sigue lógicamente, por la exfoliación de las piezas temporales, un trazo descendente en ambos grupos. Se manifiesta de igual modc; una mayor prevalencia en el grupo A, a excepción de dos elevaciones del 


\section{CUADRO 2}

Cuadro comparativo de las condiciones estudiadas en los dientes de dos grupos de niñas escolares de 7 a 13.- 350 muestras

\section{DENTICION TEMPORAL}

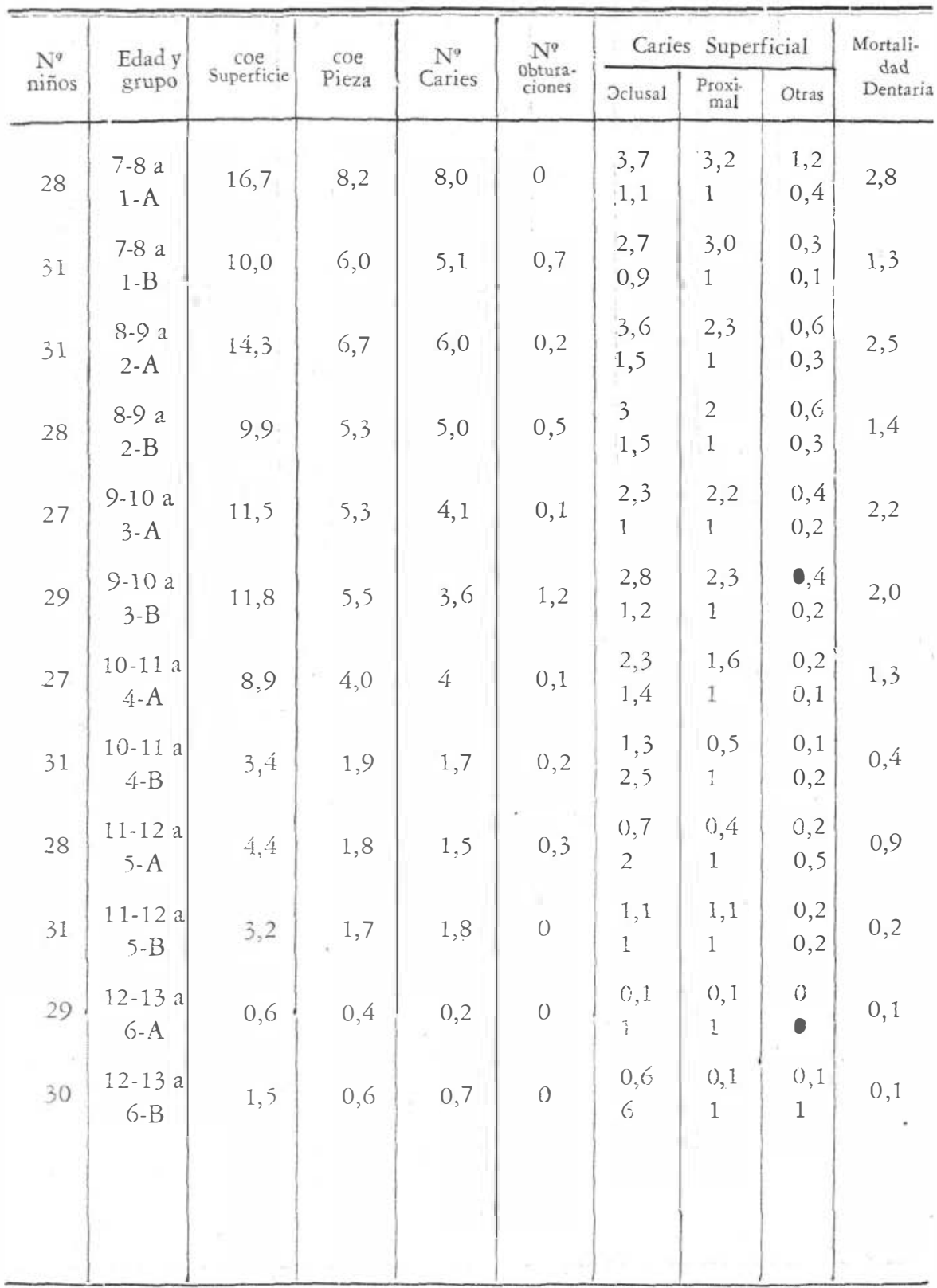




\section{CUADRO 3}

Términos promedios de las condiciones de ambos grupos en dentación permanent

\begin{tabular}{|c|c|c|c|c|c|c|c|c|c|c|c|}
\hline \multirow{2}{*}{$\underset{\text { niño }}{\mathrm{N}^{\mathrm{N}}}$} & \multirow[b]{2}{*}{ Edad } & \multirow{2}{*}{$\begin{array}{l}\text { C.O.E. } \\
\text { Super- } \\
\text { ficic }\end{array}$} & \multirow{2}{*}{$\begin{array}{c}\text { C.O.E. } \\
\text { Pieza }\end{array}$} & \multirow{2}{*}{$\underset{\text { Caries }}{\mathrm{N}^{0}}$} & \multirow{2}{*}{$\begin{array}{c}\mathrm{N}^{\mathrm{N}^{\prime}} \\
\text { cionra- } \\
\text { ciones }\end{array}$} & \multirow{2}{*}{$\begin{array}{l}\text { Salud } \\
\text { dental }\end{array}$} & \multirow{2}{*}{$\begin{array}{l}\text { Morta- } \\
\text { lidad } \\
\text { dentaria }\end{array}$} & \multicolumn{3}{|c|}{ Caries superficial } & \multirow{2}{*}{$\begin{array}{l}\text { Indice } \\
\text { affec } \\
\text { ciim } \\
\text { primer } \\
\text { molar }\end{array}$} \\
\hline & & & & & & & & $\begin{array}{c}\text { Oclu- } \\
\text { sal }\end{array}$ & $\begin{array}{c}\text { Proxi- } \\
\text { mal }\end{array}$ & Otras & \\
\hline 59 & 7- $8 a$ & 5,9 & 3,5 & 5,5 & 0,5 & $\begin{array}{c}2 \\
0 / n\end{array}$ & $\begin{array}{c}2 \\
0 / n\end{array}$ & $\begin{array}{l}4,2 \\
21\end{array}$ & $\begin{array}{c}0,2 \\
1\end{array}$ & $\begin{array}{c}1,4 \\
7\end{array}$ & 3,2 \\
\hline 59 & 8- $9 a$ & 7,4 & 4,1 & 6,5 & 0,2 & $\begin{array}{c}14 \\
0,2 / n\end{array}$ & $\begin{array}{c}14 \\
0,2 / \mathrm{n}\end{array}$ & $\begin{array}{c}4,6 \\
5\end{array}$ & $\begin{array}{c}1,1 \\
1\end{array}$ & $\begin{array}{r}1,7 \\
1\end{array}$ & 3,6 \\
\hline 56 & $9-10 \mathrm{a}$ & 8,4 & 4,9 & 6,3 & 1,0 & $\begin{array}{c}9 \\
0,2 / n\end{array}$ & $\begin{array}{c}9 \\
0,2 / \mathrm{n}\end{array}$ & $\begin{array}{c}5,2 \\
5\end{array}$ & $\begin{array}{c}1,2 \\
1\end{array}$ & $\begin{array}{c}1,7 \\
1\end{array}$ & 3,5 \\
\hline 58 & $10-11 \mathrm{a}$ & 9,7 & 6,6 & 7,9 & 0,9 & $\begin{array}{c}18 \\
0,3 / n\end{array}$ & $\begin{array}{c}21 \\
0,3 / \mathrm{n}\end{array}$ & $\begin{array}{l}6,1 \\
12\end{array}$ & $\begin{array}{l}1,9 \\
1\end{array}$ & $\begin{array}{l}1,4 \\
0,5\end{array}$ & 3,8 \\
\hline 59 & $11-12 \mathrm{a}$ & 13,5 & 8,3 & 9,2 & 1,9 & $\begin{array}{c}19 \\
0,3 / \mathrm{n}\end{array}$ & $\begin{array}{c}20 \\
0,3 / \mathrm{n}\end{array}$ & $\begin{array}{l}7,4 \\
2,5\end{array}$ & $\begin{array}{c}2,8 \\
1\end{array}$ & $\begin{array}{l}1,9 \\
0,7\end{array}$ & 3,7 \\
\hline 59 & $12-13 a$ & 15,7 & 9,9 & 11,4 & 1,7 & $\begin{array}{c}31 \\
0,5 / \mathrm{n}\end{array}$ & $\begin{array}{c}43 \\
0,7 / \mathrm{n}\end{array}$ & $\begin{array}{l}8,5 \\
3,5\end{array}$ & $\begin{array}{c}2,6 \\
1\end{array}$ & $\begin{array}{c}2 \\
0,8\end{array}$ & 3,6 \\
\hline
\end{tabular}

\section{CUADRO 4}

Términos promedios de las condiciones de ambos grupos en dentición temporal

\begin{tabular}{|c|c|c|c|c|c|c|c|c|c|}
\hline \multirow{2}{*}{$\begin{array}{c}\mathrm{N}^{\circ} \\
\text { niños }\end{array}$} & \multirow{2}{*}{ Edad } & \multirow{2}{*}{$\begin{array}{c}\text { coe } \\
\text { superficie }\end{array}$} & \multirow{2}{*}{$\begin{array}{l}c o \epsilon \\
\text { pieza }\end{array}$} & \multirow{2}{*}{$\mathrm{N}_{\text {Caries }}^{\mathrm{j}}$} & \multirow{2}{*}{$\begin{array}{l}\mathrm{N}^{\prime \prime} \\
\text { Obtura- } \\
\text { ciones }\end{array}$} & \multicolumn{3}{|c|}{ Caries Superficial } & \multirow{2}{*}{$\begin{array}{l}\text { Mortali- } \\
\text { dad } \\
\text { Dentaria }\end{array}$} \\
\hline & & & & & & Oclusal & $\begin{array}{c}\text { Proxi- } \\
\text { mal }\end{array}$ & Otras & \\
\hline 59 & 7- $8 a$ & 18,3 & 7,1 & 6,5 & 0,3 & $\begin{array}{c}3,2 \\
1\end{array}$ & $\begin{array}{c}3,1 \\
1\end{array}$ & $\begin{array}{l}0,7 \\
0,2\end{array}$ & 2,0 \\
\hline 59 & 8- $9 a$ & 12,1 & 6,0 & 5,5 & 0,3 & $\begin{array}{l}3,3 \\
1,5\end{array}$ & $\begin{array}{c}2,1 \\
1\end{array}$ & $\begin{array}{l}0,6 \\
0,3\end{array}$ & 1,9 \\
\hline 56 & $9-10$ a & $11 ; 6$ & 5,4 & 3,8 & 1,1 & $\begin{array}{l}2,5 \\
1,1\end{array}$ & $\begin{array}{c}2,2 \\
1\end{array}$ & $\begin{array}{l}0,4 \\
0,2\end{array}$ & 2,1 \\
\hline 58 & $10-11 a$ & 6,1 & 2,9 & 2,8 & 0,1 & $\begin{array}{c}1,8 \\
2\end{array}$ & $\begin{array}{c}1,0 \\
1\end{array}$ & $\begin{array}{l}0,1 \\
0,1\end{array}$ & 0,8 \\
\hline 59 & $11-12$ a & 3,8 & 1,7 & 1,6 & 0,1 & $\begin{array}{l}0,9 \\
1,3\end{array}$ & $\begin{array}{c}0,7 \\
1\end{array}$ & $\begin{array}{l}0,2 \\
0,3\end{array}$ & 0,5 \\
\hline 59 & $12-13 a$ & 1,0 & 0,5 & 0,4 & 0 & $\begin{array}{c}0,3 \\
3\end{array}$ & $\begin{array}{c}0,1 \\
1\end{array}$ & $\begin{array}{r}2,0 \\
20\end{array}$ & 0,1 \\
\hline
\end{tabular}


grupo B, entre los 9 y los 10 años, y entre los 11 y los 12, que puede deberse a tres causas:

1. Una mala interpretación en el grupo A, entre las piezas exfoliadas o extraídas.

$2^{3}$ Por un promedio mayor de piezas temporales en las niñas del grupo B, debido a una mayor mortalidad dentaria temporal del grupo A. de muestras.

3: Por simple accidente del grupo, considerando el reducido número GRÁFICA 2 :

COE y coe por pieza, promedio de ambos grupos. Puede observarse como dato de interés, que en la dentición permanente existe un marcado aumento del índice, de los nueve y medio a los diez y medio años, lo cual se explica lógicamente, por la aparición de nuevas piezas permanentes, y por ser una época de mayor susceptibilidad en el niño (5). De igual modo, hay un descenso rápido en la dentición temporal, por las piezas que se han exfoliado en la misma época. Estos índices pueden considerarse altos, si se comparan con una serie de los mismos efectuados en los Estados Unidos: (Hagerstown, Nicollet Country, Baltimore), (Newburgh-Kingston) (1), (Evaston) (11), etc. Esto se explica por el mayor contenido de carbohidratos en la dieta del niño costarricense y una menor educación en Salud Dental. Llama la atención el hecho de que en el estudio efectuado por el Departamento de Higiene Dental del Ministerio de Salubridad Pública de Costa Rica, el índice es aproximadamente dos puntos menor en todas las edades, que en este caso; es asímismo, más bajo que el efectuado en Grand Rapids en 1945 antes de la fluorinación del agua de consumo (17). Por el contrario, en el presente estudio, el índice es mayor que el de Grand Rapids, llegándose a nivelar ambas gráficas a la edad de trece años. La divergencia de esta investigación, con la efectuada por dicho Ministeric, puede deberse a otro procedimiento empleado en la tabulación de las muestras, o bien, a las dificiles circunstancias en que en el último caso fueron examinados los niños escolares (17).

\section{GRÁFICA 3:}

COE y coe por superficie, comparativo entre los grupos A y B. Lógica. mente, al considerar estos índices por superficies, tienen que expresar cif ras más altas. La comparación entre ambos grupos presenta las mismas variaciones que en los índices por pieza, sólo que en mayor escala. Puede notarse que él ascenso de la curva de la dentición permanente del grupo B es irregular, en tara to que la del grupo A es continua, lo cual indica una condición permanente por las mismas circunstancias expuestas en la Gráfica 1. 

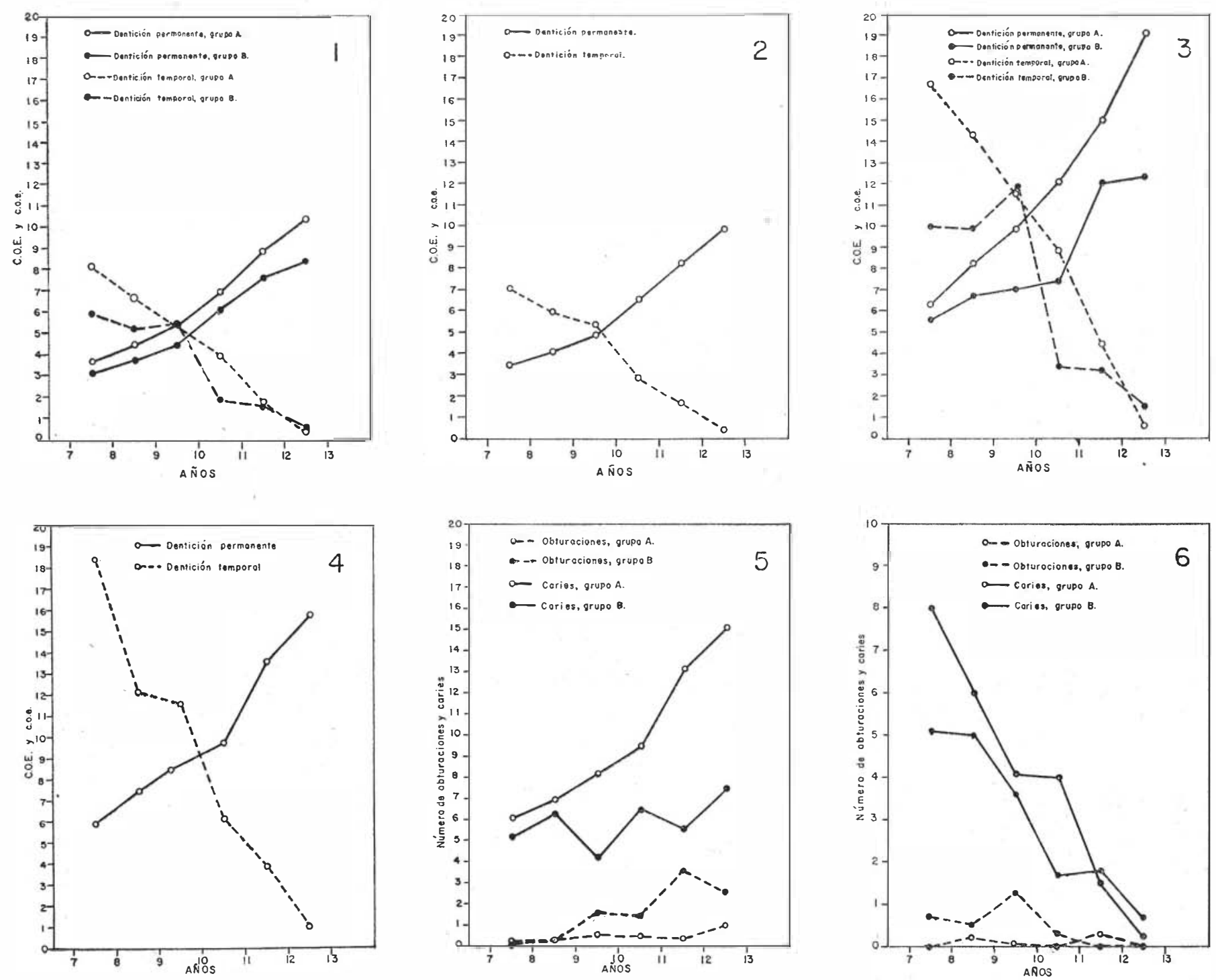
GRÁFICA 4:

COE y ioe por superficie, promedio de ambos grupos. No amerita mayor discusión, y puede apreciarse el ascenso rápido del índice COE despues de los diez años y medio, así como el descenso del coe después de los nueve y medio, ambas condiciones, por los motivos expuestos en la Gráfica 2.

\section{GRÁF!CA 5:}

Número de obturaciones y número de caries en la dentición permanente, comparativo entre ambos grupos. En ella se manifiesta claramente, la escasa atención dental que reciben las niñas del grupo A, ya sea por la condición económica o por falta de educación en ese sentido. Del estudio de las curvas se deduce, que en el grupo B, el 25 por ciento de las piezas cariadas, se encuentran obturadas, en tanto que en el grupo $A$, sólo alcanzan un 5,3 por ciento.

GRÁFICA 6:

Número de obturaciones y número de caries en la dentición temporad, comparativo entre los grupos A y B. Lo mismo que en la gráfica anterior, se nota una considerable diferencia en el porcentaje de las piezas obturadas: en el grupo $A$ es prácticamente menor que el 1 por ciento tanto que en grupo $\mathrm{B}$ asciende al 12,5 por ciento. Ambos porcentajes son muy inferiores a los de la dentición permanente (Gráfica 5), lo cual indica una vez más, la poca importancia que aún se le concede a la dentición temporal en el medio costarricense.

GRÁFICA 7:

Número de obturaciones y número de caries en las denticiones temporal y permanente; promedio de ambos grupos. De igual modo, por las proporciones de las curvas, manifiesta que se le concede mayor importancia al tratamiento operatorio de los dientes permanentes.

GRÁFICA 8:

Obtenida del estudio de la gráfica anterior, indica el porcentaje de las piezas cariadas, en relación con las obturadas, considerando el número promedio de piezas dentales en cada una de las edades especificadas en la cráfica. Es un promedio de ambos grupos. Se nota que entre los siete y medio y los nueve y medio años, es menor el porcentaje en la dentición temporal, lo cual sc explica por dos motivos:

a) Por el elevado porcentaje de mortalidad dentaria temporal, que reduce el número de caries. 
b) Por la menor cantidad de piezas permanentes erupcionadas.

Por otro lado, puede apreciarse, como en términos generales, despué: de los nueve años y medio, se comienza a dispensarle un mayor interés y po: consecuencia, atención a la dentición permanente.

\section{GRÁFICA 9:}

Indices de salud dental, afección del primer molar permanente y mortalidad dentaria temporal, comparativo entre ambos grupos. Las tres condiciones se discutirán en la gráfica siguiente, restando por mencionar en ésta, que siempre existe una marcada superioridad en el grupo B, en cualquier afección que se considere. Los trazos de las tres condiciones son prácticamente paralelos, aunque vuelve a notarse un accidente de grupo, en la elevación de la mortalidad dentaria temporal del grupo B entre los 9 y los 10 años, que en el caso concreto, no sobrepasa sin embargo, a la del grupo A. El índice de la salud dental se acentúa progresivamente en el grupo A, lo cual indica negligencia en el tratamiento de los primeros molares permanentes, que afectados desde una temprana edad, llegan finalmente a perderse.

GRÁFICA 10:

Indices de salud dental, afección del primer molar permanente y mortalidad dentaria temporal; promedio de ambos grupos. El índice de salud dental se manifiesta en una forma acumulativa o progresiva, y si se quiere, toma proporciones significantes, considerando que a la edad de trece años se ha perdido media molar permanente por niña. El índice de afección de esta pieza, muestra un trazo, que aunque irregular, dentro de las cifras comprendidas, puede interpretarse como una línea recta; ello indica, que en vez de considerar un aumento progresivo de la lesión, puede suponerse que ella se manifiesta en sus primeros años. Siempre existe un porcentaje de primeros molares, que aunque muy reducido, no llegan a cariarse, como lo demuestra la gráfica, que en ningún grupo, alcanza el punto óptimo de 4. Aún cuando el número de las muestras sea reducido, y las variaciones en la curva indiquen accidentes de los grupos, los resultados son similares a los obtenidos por CRINER y LEÓN-LARA (7). Forzosamente la curva de la mortalidad dentaria temporal va en descenso, más acentuada entre los diez y medio y once y medio años, por la pérdida natural de dichas piezas.

\section{CuAdro 5:}

Cuadro comparativo entre ambos grupos de la condición de la boca. La mayoría de las condiciones favorables recaen dentro del grupo B. Por otro lado, aún cuando dichas condiciones se han clasificado en una forma arbitraria, puede 

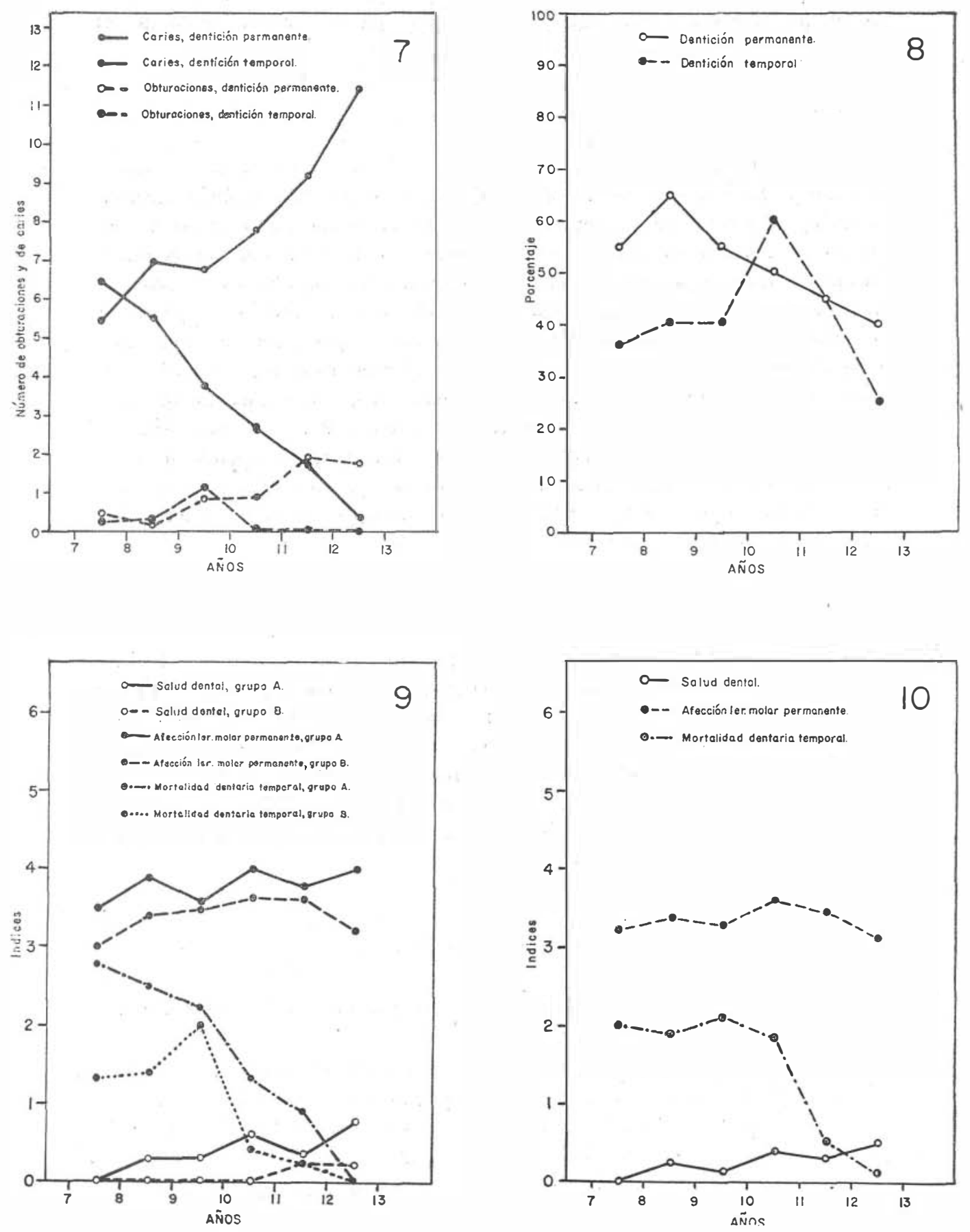
notarse que la mayoría se encuentran en el tipo regular, a lo cual, sumados los porcentajes de bocas malas y pésimas, se deduce que un 72 por ciento de las niñas requieren un tratamiento urgente.

\section{Cuadro 6:}

Cuadro comparativo entre ambos grupos, de la frecuencia de las visitas al dentista. La totalidad de las niñas del grupo $\mathrm{B}$ que han recibido atención de su boca, ha sido con un dentista privado, en tanto que en el grupo A, aún cuando un número reducido de ellas recurren donde el mismo, por la información obtenida, pudo comprobarse que frecuentan los consultorios de prácticos dentales o "empíricos", cuyas tarifas son ridículas y su servicio se reduce en su mayoría, a prácticas de exodoncia. Si bien un 34 por ciento de niñas aparecen visitando al dentista por rutina, se dijo anteriormente que no puede considerarse estrictamente en esa forma, sino más bien, como aquellas que han sido llevadas donde el mismo en repetidas ocasiones. Por otro lado, entre el grupo de niñas que nunca lo han visitado, una cuarta parte corresponde al grupo $\mathrm{B}$, y el porcentaje de todas ellas, sumado al de las que lo visitan por emergencia, es de un 66 por ciento, e indica que está muy poco extendida la educación dental en las familias costarricenses.

\section{CUADRO 7:}

Cuadro comparativo entre ambos grupos, de los recursos para la higiene de la boca. En su totalidad, las niñas del grupo B poseen cepillo y dentífrico siendo relativamente bajos los porcentajes de las del grupo A que no lo poseen, ya sea por pobreza o por negligencia. Sin embargo, por las condiciones estudiadas en la boca, necesariamente más acentuadas en el grupo A, se deduce que el problema radica fundamentalmente, en que las niñas no hacen uso rutinario de dichos implementos, o bien, por la no aplicación de métodos correctos en el cepillado de los dientes.

\section{CONCLUSIONES}

Las conclusiones obtenidas en esta investigación pueden resumirse del siguiente modo:

1. Debe reconocerse ante todo, que la condición económica y el nivel de educación, juegan un papel de gran importancia en el estado de la boca de los niños, pues en el medio costarricense, esos factores influyen sobre la higiene, un mayor o menor consumo de carbohidratos y la educación dental.

2. El estudio realizado, demuestra una mayor necesidad de educación dental y atención de la boca en las niñas del tipo del grupo A. Sin embargo, la educación dental debe de hacerse en una forma generalizada, pues aún cuan- 
CUADRO 5

Cuadro comparativo, entre ambos grupos, de la condición de la boca

\begin{tabular}{|c|c|c|c|c|c|}
\hline \multirow{2}{*}{$\begin{array}{l}\text { Edad y } \\
\text { grup }\end{array}$} & \multicolumn{5}{|c|}{ Condición de la boca } \\
\hline & Buena & $\begin{array}{l}\text { Acep } \\
\text { table }\end{array}$ & Regu- & Mala & Pésima \\
\hline $\begin{array}{c}7-8 a \\
1-A\end{array}$ & 1 & 2 & 7 & 14 & 4 \\
\hline $\begin{array}{c}7.8 \mathrm{a} \\
1-\mathrm{B}\end{array}$ & 4 & 4 & 14 & 9 & 0 \\
\hline $\begin{array}{c}8-9 a \\
2-A\end{array}$ & () & 3 & 6 & 14 & 8 \\
\hline $\begin{array}{c}8-9 \text { a } \\
2-B\end{array}$ & 3 & 5 & 11 & 9 & 0 \\
\hline $\begin{array}{c}9-10 a \\
3-A\end{array}$ & 0 & 3 & 12 & 13 & - \\
\hline $\begin{array}{c}9-10 \text { a } \\
3-B\end{array}$ & 1 & 9 & 12 & 8 & 0 \\
\hline $\begin{array}{c}10-11 \mathrm{a} \\
4-\mathrm{A}\end{array}$ & 0 & 2 & 10 & 11 & 4 \\
\hline $\begin{array}{c}10-11 \mathrm{a} \\
4-\mathrm{B}\end{array}$ & 2 & 18 & 8 & 3 & 0 \\
\hline $\begin{array}{c}11-12 \mathrm{a} \\
5-\mathrm{A}\end{array}$ & 0 & 5 & 12 & 10 & 1 \\
\hline $\begin{array}{c}11-12 a \\
5-B\end{array}$ & 2 & 12 & 12 & 4 & 1 \\
\hline $\begin{array}{c}12-13 \mathrm{a} \\
6-\mathrm{A}\end{array}$ & 0 & 5 & 18 & 3 & 3 \\
\hline $\begin{array}{c}12-13 a \\
6-B\end{array}$ & 3 & 13 & 13 & 1 & 0 \\
\hline TOTAL & 16 & 81 & 136 & 96 & 21 \\
\hline $\begin{array}{l}\text { Porcen- } \\
\text { taje }\end{array}$ & $5 \%$ & $23 \%$ & $39 \%$ & $27 \%$ & $6 \%$ \\
\hline
\end{tabular}

CUADRO 6

Cuadro comparativo, entre ambos grupos, de la frecuencia de las visitas al dentista

\begin{tabular}{|c|c|c|c|c|c|}
\hline \multirow{2}{*}{$\begin{array}{l}\text { Edad y } \\
\text { grupo }\end{array}$} & \multicolumn{2}{|c|}{ Privado } & \multicolumn{2}{|c|}{$\begin{array}{l}\text { Dispensario } \\
\text { Público }\end{array}$} & \multirow{2}{*}{$\underset{c a}{N u n}$} \\
\hline & $\begin{array}{l}\text { Emer- } \\
\text { gencia }\end{array}$ & $\begin{array}{l}\text { Ruti- } \\
\text { naria }\end{array}$ & \begin{tabular}{|l|}
$\begin{array}{c}\text { Emer- } \\
\text { gencia }\end{array}$ \\
\end{tabular} & $\begin{array}{l}\text { Ruti- } \\
\text { naria }\end{array}$ & \\
\hline $\begin{array}{c}7-8 a \\
1-A\end{array}$ & 6 & 0 & 7 & 0 & 15 \\
\hline $\begin{array}{c}7-8 a \\
1-B\end{array}$ & 10 & 10 & 0 & 0 & 11 \\
\hline $\begin{array}{l}8-9 a \\
2-A\end{array}$ & 3 & 0 & 9 & 0 & 18 \\
\hline $\begin{array}{c}8-9 a \\
2-B\end{array}$ & 11 & 15 & 0 & 0 & 3 \\
\hline $\begin{array}{c}9-10 \mathrm{a} \\
3-\mathrm{A}\end{array}$ & 6 & 2 & 8 & 0 & 11 \\
\hline $\begin{array}{c}9-10 \text { a } \\
3-B\end{array}$ & 4 & 13 & 0 & 0 & 2 \\
\hline $\begin{array}{c}10-11 \text { a } \\
4-A\end{array}$ & 12 & 2 & 5 & 2 & 6 \\
\hline $\begin{array}{c}10-11 \text { a } \\
4-B\end{array}$ & 6 & 23 & 0 & 0 & 2 \\
\hline $\begin{array}{c}11-12 a \\
5-A\end{array}$ & 8 & 0 & 12 & 0 & 8 \\
\hline $\begin{array}{c}11-12 a \\
5-B\end{array}$ & 4 & 24 & 0 & 0 & 3 \\
\hline $\begin{array}{c}12-13 \mathrm{a} \\
6-\mathrm{A}\end{array}$ & 7 & 3 & 10 & 1 & 8 \\
\hline $\begin{array}{c}12-13 \mathrm{a} \\
6-\mathrm{B}\end{array}$ & 26 & 0 & 0 & 2 & 2 \\
\hline TOTAL & 113 & 92 & 51 & 6 & 88 \\
\hline $\begin{array}{l}\text { Porcen- } \\
\text { taje }\end{array}$ & $32 \%$ & $26 r$ & $15 \%$ & $2 \%$ & $25 \%, c$ \\
\hline
\end{tabular}




\section{CUADRO 7}

Cuadro comparalivo entre ambos grupos de los recursos para la bigiene de la bocu

\begin{tabular}{|c|c|c|c|c|c|c|}
\hline \multirow{3}{*}{$\begin{array}{l}\text { Edad y } \\
\text { grupo }\end{array}$} & \multicolumn{3}{|c|}{ Poseen cepillo } & \multicolumn{3}{|c|}{ Poseen Dentífrico } \\
\hline & \multirow{2}{*}{ Sí } & \multicolumn{2}{|c|}{ No } & \multirow{2}{*}{ Sí } & \multicolumn{2}{|c|}{ No } \\
\hline & & Pobreza & $\begin{array}{c}\text { Negligen } \\
\text { cia }\end{array}$ & & Pobreza & $\begin{array}{c}\text { Negligen } \\
\mathrm{Cia}\end{array}$ \\
\hline $\begin{array}{c}7-8 \text { a. } \\
1-A\end{array}$ & 23 & 1 & 4 & 21 & 1 & 6 \\
\hline $\begin{array}{c}7-8 \mathrm{a} . \\
1-\mathrm{B}\end{array}$ & 31 & 0 & 0 & 31 & 0 & 0 \\
\hline $\begin{array}{c}8-9 \text { a. } \\
2-A\end{array}$ & 27 & 2 & 2 & 26 & 2 & 3 \\
\hline $\begin{array}{c}8-9 \text { a. } \\
2-B\end{array}$ & 28 & 0 & 0 & 28 & 0 & 0 \\
\hline $\begin{array}{c}9-10 \text { a. } \\
3-A\end{array}$ & 25 & 1 & 1 & 23 & 2 & 2 \\
\hline $\begin{array}{c}9-10 \mathrm{a} . \\
3-\mathrm{B}\end{array}$ & 29 & 0 & 0 & 29 & 0 & 0 \\
\hline $\begin{array}{c}10-11 \text { a. } \\
4-A\end{array}$ & 26 & 1 & 0 & 26 & 1 & 0 \\
\hline $\begin{array}{c}10-11 \mathrm{a} . \\
4-\mathrm{B}\end{array}$ & 31 & 0 & 0 & 31 & 0 & 0 \\
\hline $\begin{array}{c}11-12 \mathrm{a} . \\
5-\mathrm{A}\end{array}$ & 28 & 0 & 0 & 25 & 3 & 0 \\
\hline $\begin{array}{c}11-12 \text { a. } \\
5-B\end{array}$ & 31 & 0 & 0 & 31 & 0 & 0 \\
\hline $\begin{array}{c}12-13 \text { a } \\
6-A\end{array}$ & 29 & 0 & 0 & 28 & 0 & 1 \\
\hline $\begin{array}{c}12-13 \text { a } \\
\text { 6-B }\end{array}$ & 30 & 0 & 0 & 30 & 0 & 0 \\
\hline TOTAL & 338 & 5 & 7 & 329 & 12 & 12 \\
\hline Porcentaje & $97 \%$ & $1 C / c$ & $2 \%$ & $94 \%$ & $3 C_{j}^{1} \mathrm{C}$ & $3 C / c$ \\
\hline
\end{tabular}


do las niñas del grupo B presentan mejores condiciones en su boca, no pueden considerarse muy favorables.

3. La labor educacional debe hacerse principalmente en los centros de enseñanza, no sólo escolares, sino pre-escolares. Debe impartirse ante todo, una instrucción adecuada a los maestros, a efecto de que pongan los principios básicos en conocimiento, no sólo de los niños, sino también de los padres de familia. Esto debe complementarse con material educativo, por medio de láminas ilustrativas cuyos contenidos estén al nivel del entendimiento del niño, exhibidos en las aulas y otros sitios de las escuelas más frecuentados por los alumnos. Buenos complementos para ello serían también, publicaciones periódicas por la prensa y conferencias radiales.

4. Los puntos básicos que debe abarcar este aspecto de educación deben ser:

a) El control estricto sobre el consumo de carbohidratos refinados, ya que es un hecho comprobado y aceptado, que en ello radica el factor etiológico determinante de las caries (4) (12) (13) (17) (18). Este control es, sin embargo, un problema de considerarble magnitud en el niño, y debe de recurrirse a medidas más apropiadas, que aunque no efectivas en un ciento por ciento cuando menos producirán una reducción apreciable en los índices de caries. Entre ellas pueden considerarse, el evitar el consumo en horas escolares, ya que el azúcar es más perjudicial entre las comidas, que junto con ellas (19), así como, determinar cuáles alimentos, tanto por su consistencia como por su contenido de azúcar, son los más nocivos (20), a efecto de proscribirlos y recomendar otros de mayor valor nutritivo.

El consumo mínimo de alimentos azucarados junto con las comidas, deberá ser recomendado. Sin embargo, como ya se dijo, en muchos niños costarricenses, el "agua dulce" y el café por condición económica, llegan a ser los sustitutos de la leche, por lo cual se hace recesaria una estrecha colaboración entre las autoridades sanitarias y educacionales, a efecto de proveer de tan valioso alimento a esos niños en sus horas escolares.

b) Una instrucción adecuada sobre la higiene de la boca y el cepillado de los dientes. Deberá insistirse, tanto en una técnica apropiada (21), así como en el tipo de cepillo adecuado (de fibra natural, dos hileras de penachos, puño corto y recto), ya que en el estudio pudo observarse que en esos dos factores, radican fundamentalmente la deficiencia en la higiene de la boca. Deberá proporcionárseles cepillo y dentífrico a aquellos niños que por pobreza no puedan adquirirlos.

c) Insistir en la visita rutinaria donde el dentista, ya sea al consultorio privado o al dispensario público, según la condición económica de los padres. Desde el punto de vista de la Salud Pública Dental, deberá recomendarse la visita dos veces al año, aún cuando en la práctica privada sea un asunto muy particular de cada caso (10). La atención dental del niño pobre se torna otro problema, por la falta de dispensarios públicos que llenen sus necesidades en una forma 
efectiva. Por consiguiente, al elaborar los presupuestos de sanidad, es conveniente contemplar a fondo este asunto, considerando en su favor, que el número de dentistas en Costa Rica aumenta año a año, cuyos servicios pueden ser utilizados en este aspecto.

d) Dar a conocer los factores etiológicos que ocasionan malas oclusiones, y las medidas más apropiadas para prevenirlas.

5. La alta mortalidad dentaria temporal y el bajo porcentaje de piezas tratadas, indican la necesidad de continuar insistiendo en el medio costarricense, sobre la importancia que encierra dicha dentición.

6. La alta incidencia de la afección del primer molar, indica también, la conveniencia de optar siempre por medidas profilácticas preventivas para la preservación de dicha pieza, practicando desde una temprana edad, la eliminación de sus fisuras con la preparación de una cavidad y su obturación con un material permanente.

7. El estudio indica que existe en los niños costarricenses, una inmunidad a caries de aproximadamente un 1 por ciento ( 4 muestras con COE: 0 ), y una inmunidad relativa de un 3 por ciento ( 9 muestras con COE: 1 y 2 ). Ello no implica necesariarinente que no consuman azúcar, pues lo acusan en su dieta, sino a factores residentes en la saliva (12). El único caso con el azúcar restringido fü el de una niña diabética (COE: 1 ), lo cual comprueba su papel en la producción de caries.

\section{RESUMEN}

1. Con el fin de obtener bases concretas para una efectiva educación dental en los niños costarricenses, el Departamento de Odontología Infantil y Preventiva de la Facultad de Odontología de la Universidad de Costa Rica, ha iniciado un estudio estadístico sobre caries y otras condiciones de la boca, en las niñas escolares de San José.

2. Este trabajo comprende la primera parte de la investigación, o sea el de la parte femenina, en el cual se ha hecho un estudio comparativo entre dos grupos de niñas escolares de diferente nivel económico y social, las cuales se denominaron grupo A y grupo B, con un total de 349 muestras.

3. El estudio efectuado por medio de interrogaciones y exámenes clín.cos. abarcó los siguientes aspectos:

I. Condiciones generales:

A. Estado de la boca.

B. Consumo de azúcar.

C. Frecuencia de las visitas al dentista.

D. Higiene de la boca. 
II. Condiciones de los dientes:

A. Dentición permanente:

a. COE por pieza y por superficies.

b. Número de caries.

c. Nímero de obturaciones.

d. Indice de salud dental.

e. Indice de mortalidad dentaria.

f. Relación entre caries oclusales, proximales y de otros tipos.

g. Indice de afección del primer molar.

B. Dentición temporal:
a. coe por pieza y por superficies.
b. Número de caries.
c. Número de obturaciones.
d. Relación entre carics oclusales, proximales y de otros tipos.
e. Mortalidad dentaria.

4. Los resultados se han clasificado en siete cuadros y en diez gráficas, y de muestran una superioridad de condiciones en todo sentido en las niñas del grupo B.

\section{SUMMMRY}

1. So as to obtain solid bases for a n effective dental education of costarican children, the Department of Infantile and Preventive Odontology of the Faculty of Odontology of the University of Costa Rica has begun a statistical study of caries and other oral conditions among school children of San José.

2. This paper represents the first part of the study, the female cases. It deals on a comparative analysis between two groups of school girls of different economic and social level, designated as Group A and Group B, and comprising a total of 349 samples.

3. The investigation was carried out by means of questionings and clinical examinations, and contemplated the following points:

I. General conditions:
A. Oral conditions.
B. Sugar consumption.
C. Frequency of dental examinations.
D. Oral hygiene. 
II. Condition of the teeth.

A. Permanent dentition.

a. DMF for each tooth and surface.

b. Number of caries.

c. Number of obturations.

d. Dental health index.

e. Dental mortality index.

f. Relation among occlusated, proximal, and other types of caries.

g. Index of affection of the first molar.

B. Deciduos dentition:

a. dmf for each tooth and surface.

b. Number of caries.

c. Number of obturations.

d. Relation among occlusal, proximal, and other types of caries.

e. Dental mortality.

4. The results are presented in seven tables and ten graphs, and show a manifest superiority of conditions in every respect among the girls of Group B.

\section{BIBLIOGRAFIA}

1. Ast, D. B.

1955. The Newburgh-Kingston caries fluorine study. VII. Correlation of ingested water fluorides to dentofacial development. Amer. J. Ortbodont. 41:45-54.

2. Bavetta, L. A.

1955. Sugar-natural or refined. South Calif. S. Dent. Ass. J. 23:21-5.

3. BECKS, H.

1950. Carbohydrate restriction in the prevention of clental caries using the L. a. count as one index. Calif. S. Dent. Ass. J. 26:53-8.

4. BIBBY, B. G.

1951. Evaluation of caries-producing potentialities of various foodstuffs. J. Amer. dent Ass. 42 (5):491-509.

5. Brauer, J. C. et al.

1952. Dentistry for children, 3rd. ed., XII + 454 pp. Blackinston, New York

6. Brown, W. E.

1952. The utilization of radiology in a children's practice. N. S. J. Dent. Soc.. $23: 17-27$.

7. Criner, C. \& F. León-Lara

1955. Indice de afectación del primer molar permanente en los niños, sus posibles causas y prevención. Odont. Infant., 6-7:7-24. 
8. Dollar, M. L. \& H. Kulstad

1949. The economical aspect of the dental bealth problem. (p. 25-44). En PeLton, W. J. \& J. M. WISAN, Dentistry in Public Health. XI + 363 pp. Saunders, Philadelphia, Pa.

9. EASLICK, K. A.

1948. Dental caries: merbanism and present control technics. 234 pp. Mosby, Ed. St. Louis.

10. EASLICK, K. A.

1953. Comunicación personal.

11. Hill, I. N., J. R. Blayney \& W. WolF

1955. The Evanston dental caries study. XI. The caries experience rate of 12-, 13-, and 14 year old children after exposure to fluoridated water for fifty nine to seventy months. J. Dent. Ress., 34:77-87.

12. НоGевоOм, F. E.

1953. Practical pedodontia or juvenile operative dentistry and public bealth dentistry. Gth ed. 642 pp. Moshy, F. St. Louis.

13. Hurtarte, A. \& N. S. Scrimshaw

1955. Dental findings in a nutritional study of school children in five Guatemalan highland villages. J. Dent. Res., 43:390-96.

14. JAY, P.

1953. Comunicación personal.

15. Johnson, M.

1955. Comunicación personal.

16. KNutson, J. M.

1949. Surveys and the examination of dental programs. (p.6-91). En PELTON W. J. \& J. M. WIsAN, Dentistry in l'ublic Health. XI + 363 pp. Saunders, Philadelphia, $\mathrm{Pa}$.

17. LACHNER, R.

1955. Estudio sobre la prevalencia de caries dental en grupos escolares. Salud. $2: 8-12$.

18. Pauly, R.

1954. Caries dentaria: diagnóstico bacteriológico y control por la reducción de azúcares. (p. 515-25). En Memoria del IV Congreso Nacional 986 pp. Gráficos Universo, La Habana.

19. Pauly, R.

1955. Contribución al estudio de la caries dentaria en niños custarricenses. Rev. Biol. Trop., 3 (1):79-94.

20. Pauly, R.

1956. Odontologia infantil y preventiva. 250 pp. Departamento de Publicaciones Universidad de Costa Rica. (En prensa)

21. SCRIMSHAw, N. S., et al.

1953. Health aspects for the community development project, rural area, Turrialba, Costa Rica, 1948-1951. Am. J. Trop. Med. \& Hyg. 2:583. 